\title{
Core Needle Biopsy Is a More Conclusive Follow-up Method Than Repeat Fine Needle Aspiration for Thyroid Nodules with Initially Inconclusive Results: A Systematic Review and Meta-Analysis
}

Jung-Soo Pyo · Jin Hee Sohn Guhyun Kang $^{1}$

Department of Pathology, Kangbuk Samsung Hospital, Sungkyunkwan University School of Medicine, Seoul; ' ${ }^{1}$ Department of Pathology, Inje University Sanggye Paik Hospital, Seoul, Korea

Received: January 25, 2016

Revised: February 12, 2016

Accepted: February 15, 2016

Corresponding Author

Jin Hee Sohn, MD

Department of Pathology, Kangbuk Samsung

Hospital, Sungkyunkwan University School of

Medicine, 29 Saemunan-ro, Jongno-gu, Seoul

03181, Korea

Tel: +82-2-2001-2391

Fax: +82-2-2001-2398

E-mail: jhpath.sohn@samsung.com

\begin{abstract}
Background: This study investigated the appropriate management of thyroid nodules with prior non-diagnostic or atypia of undetermined significance/follicular lesion of undetermined significance (AUS/FLUS) through a systematic review and meta-analysis. Methods: This study included 4,235 thyroid nodules from 26 eligible studies. We investigated the conclusive rate of follow-up core needle biopsy (CNB) or repeat fine needle aspiration ( $\mathrm{FFNA}$ ) after initial fine needle aspiration (FNA) with non-diagnostic or AUS/FLUS results. A diagnostic test accuracy (DTA) review was performed to determine the diagnostic role of the follow-up CNB and to calculate the area under the curve (AUC) on the summary receiver operating characteristic (SROC) curve. Results: The conclusive rates of follow-up CNB and rFNA after initial FNA were 0.879 (95\% confidence interval [Cl], 0.801 to 0.929$)$ and $0.684(95 \% \mathrm{Cl}, 0.627$ to 0.736$)$, respectively. In comparison of the odds ratios of CNB and rFNA, CNB had more frequent conclusive results than rFNA (odds ratio, 5.707; $95 \% \mathrm{Cl}, 2.530$ to 12.875). Upon subgroup analysis, follow-up CNB showed a higher conclusive rate than rFNA in both initial non-diagnostic and AUS/FLUS subgroups. In DTA review of followup CNB, the pooled sensitivity and specificity were $0.94(95 \% \mathrm{Cl}, 0.88$ to 0.97$)$ and $0.88(95 \% \mathrm{Cl}$, 0.84 to 0.91 ), respectively. The AUC for the SROC curve was 0.981 , nearing 1. Conclusions: Our results show that CNB has a higher conclusive rate than rFNA when the initial FNA produced inconclusive results. Further prospective studies with more detailed criteria are necessary before follow-up CNB can be applied in daily practice.
\end{abstract}

Key Words: Thyroid nodule; Non-diagnostic or atypia of undetermined significance/follicular lesion of undetermined significance; Follow-up core needle biopsy; Repeat fine-needle aspiration; Metaanalysis
Papillary thyroid carcinoma, which has recently increased in incidence, is the most common malignant tumor in endocrine system. ${ }^{1}$ The cause for the increased incidence of papillary thyroid carcinoma is not fully understood. ${ }^{1}$ One possible cause is the improvement in ultrasonography and computed tomography. ${ }^{2,3}$ In daily practice, the treatment and follow-up for thyroid nodules are based on the results of initial fine needle aspiration (FNA). According to current guidelines, ${ }^{4-6}$ FNA is recommended as the initially performed modality, and additional testing is suggested as indicated by the initial FNA results.

In daily practice, repeat FNA (rFNA) is recommended for thyroid nodules of non-diagnostic or atypia of undetermined significance/follicular lesion of undetermined significance (AUS/ FLUS). In addition, for definite diagnosis of thyroid nodules, $B R A F^{\mathrm{V} 600 \mathrm{E}}$ mutation test or diagnostic surgery can also be performed. In previous studies, the rates of non-diagnostic and AUS/FLUS were $5 \%-17 \%$ and $3 \%-18 \%$, respectively. ${ }^{7-9}$ For thyroid nodule with non-diagnostic result, the possibility of an inconclusive reading might also be higher with $\mathrm{rFNA}$. Although $\mathrm{rFNA}$ is recommended for non-diagnostic or AUS/FLUS thyroid nodules in the current guidelines, other modalities, such as core needle biopsy (CNB) or combination of FNA and CNB, have been introduced in recent reports. ${ }^{10,11}$ However, the effectiveness or diagnostic role of these follow-up modalities has not been fully elucidated.

In this study, follow-up CNB was defined as CNB performed after initial non-diagnostic or AUS/FLUS findings. We investigated the conclusive rates of the follow-up procedures of $\mathrm{CNB}$ and rFNA in thyroid nodule with initial non-diagnostic or AUS/ FLUS finding through a systematic review and meta-analysis. Indeed, the diagnostic test accuracy (DTA) review was performed to determine the diagnostic accuracy of follow-up CNB in thy- 
roid nodules with initial inconclusive results.

\section{MATERIALS AND METHODS}

\section{Literature search and selection criteria}

Relevant articles were obtained from a search of PubMed and MEDLINE databases through December 31, 2015. The search was performed using 'thyroid,' 'core needle biopsy', and 'fine needle aspiration' as search terms. The titles and abstracts of all returned articles were screened for exclusion. To find additional eligible studies, review articles were also screened. Search results were then reviewed and included if (1) initial FNA for a thyroid nodule was performed, and (2) there was information about CNB or FNA as a follow-up study for thyroid nodules with initial non-diagnostic or AUS/FLUS results. Articles were excluded if they were (1) non-original articles or case reports or (2) nonEnglish language publications.

\section{Data extraction}

The following information was collected from the full texts of eligible studies and verified: name of first author, publication year, study location, number of patients analyzed, and method and results of initial and follow-up studies. We did not define a minimal number of patients to be included in a study. Any disagreements were resolved by consensus.

\section{Statistical analysis}

Data were analyzed using the Comprehensive Meta-Analysis software package (Biostat, Englewood, NJ, USA). We evaluated the conclusive rates of follow-up studies with follow-up CNB or
rFNA after initial FNA with non-diagnostic or AUS/FLUS results. The conclusive results included benign, follicular neoplasm or suspicious for follicular neoplasm, suspicious for malignancy, and malignancy categories. The conclusive rates were measured by dividing the number of conclusive results into the total number of cases with a follow-up study. The heterogeneity between eligible studies was assessed using $\mathrm{Q}$ and $\mathrm{I}^{2}$ statistics and presented using p-values. A sensitivity analysis was performed to assess the impact of each study on the combined effect and the heterogeneity of eligible studies. To identify any publication bias, Egger's test and Begg's funnel plot were initially performed. When a significant publication bias was found, the fail-safe $\mathrm{N}$ and trimfill tests were additionally conducted to confirm the degree of bias. The results were considered statistically significant at $\mathrm{p}<0.05$.

A DTA review was conducted using the Meta-Disc program ver. 1.4. ${ }^{12}$ The forest plots of pooled sensitivity and specificity and the summary receiver operating characteristic (SROC) curve were determined as described previously. ${ }^{13}$ The diagnostic odds ratio $(\mathrm{OR})$ and the value of the area under the curve (AUC) on SROC were investigated.

\section{RESULTS}

\section{Selection and characteristics of studies}

In the current study, 356 reports were identified in the database search. Among the search results, 209 reports were excluded due to insufficient information. In addition, 121 reports were excluded for the following reasons: focusing on other diseases ( $\mathrm{n}=$ 86), non-original articles $(n=17)$, duplicate articles $(n=13)$, and articles in a language other than English $(n=5)$. Twenty-six eli-

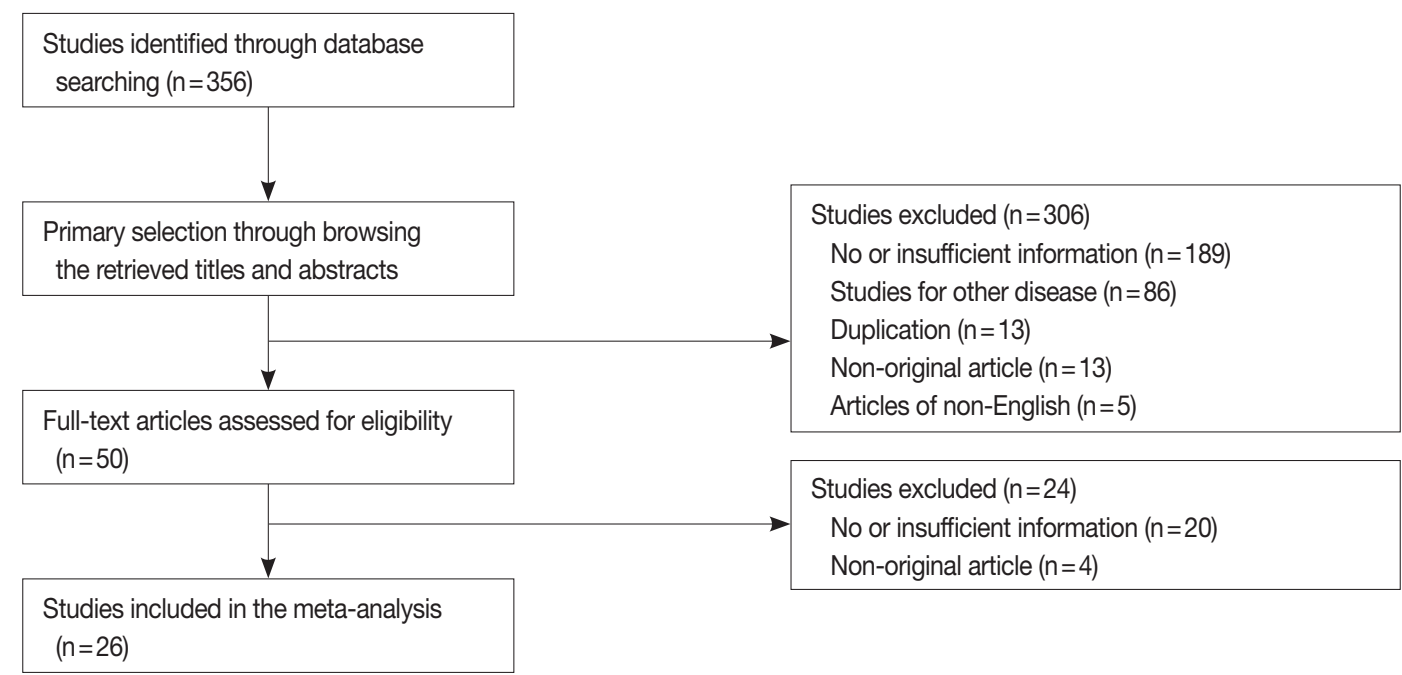

Fig. 1. Flow chart of study search and selection methods. 
gible studies and 4,253 thyroid nodules were ultimately included in the current study (Table 1, Fig. 1). ${ }^{14-39}$ The characteristics of the included studies are shown in Table 1.
Higher conclusive rate in follow-up CNB than in rFNA

For initial FNA with non-diagnostic or AUS/FLUS, followup studies using $\mathrm{CNB}$ or $\mathrm{rFNA}$ showed an overall conclusive

Table 1. Main characteristics of eligible studies

\begin{tabular}{|c|c|c|c|c|c|c|}
\hline \multirow{2}{*}{ Study } & \multirow{2}{*}{ Location } & \multirow{2}{*}{$\begin{array}{c}\text { Diagnosis of } \\
\text { initial FNA }\end{array}$} & \multirow{2}{*}{$\begin{array}{l}\text { Follow-up } \\
\text { study }\end{array}$} & \multirow{2}{*}{ No. } & \multicolumn{2}{|c|}{ Results of follow-up study } \\
\hline & & & & & Conclusive & Inconclusive \\
\hline Anderson et al..$^{14}$ (2014) & USA & Non-diagnostic & FNA & 336 & 263 & 73 \\
\hline Baloch et al..$^{15}$ (2003) & USA & Non-diagnostic & FNA & 123 & 92 & 31 \\
\hline Chen et $a l .^{16}(2012)$ & USA & AUS/FLUS & FNA & 26 & 17 & 9 \\
\hline \multirow[t]{2}{*}{ Choi et al..$^{17}$ (2014) } & Korea & Non-diagnostic & CNB & 128 & 120 & 8 \\
\hline & & & FNA & 140 & 40 & 100 \\
\hline Choi et al. ${ }^{18}$ (2014) & Korea & AUS/FLUS & CNB & 107 & 103 & 4 \\
\hline Dincer et al..$^{19}$ (2013) & Turkey & AUS/FLUS & FNA & 74 & 51 & 23 \\
\hline Gocun et al..$^{20}$ (2014) & Turkey & AUS/FLUS & FNA & 118 & 73 & 45 \\
\hline Gweon et al. ${ }^{21}$ (2013) & Korea & AUS/FLUS & FNA & 86 & 81 & 5 \\
\hline Ho et al..$^{22}$ (2014) & USA & AUS/FLUS & FNA & 116 & 74 & 42 \\
\hline Hyeon et al..23 (2014) & Korea & AUS/FLUS & FNA & 274 & 214 & 60 \\
\hline Jo et al. ${ }^{24}(2011)$ & USA & Non-diagnostic & FNA & 363 & 267 & 96 \\
\hline \multirow[t]{2}{*}{ Lee et al..$^{25}(2015)$} & Korea & AUS/FLUS & CNB & 34 & 28 & 6 \\
\hline & & & FNA & 118 & 74 & 44 \\
\hline \multirow[t]{2}{*}{ Lee et al..$^{26}$ (2014) } & Korea & Non-diagnostic & CNB & 121 & 114 & 7 \\
\hline & & & FNA & 357 & 239 & 118 \\
\hline Moon et al..$^{27}(2015)$ & Korea & AUS/FLUS & FNA & 246 & 185 & 61 \\
\hline Moslavac et al..28 (2012) & Croatia & Non-diagnostic & FNA & 38 & 31 & 7 \\
\hline \multirow[t]{2}{*}{ Na et al.. ${ }^{29}(2015)$} & Korea & AUS/FLUS & CNB & 158 & 110 & 48 \\
\hline & & & FNA & 158 & 75 & 83 \\
\hline \multirow[t]{4}{*}{ Na et al. ${ }^{30}$ (2012) } & Korea & Non-diagnostic & CNB & 45 & 43 & 2 \\
\hline & & & FNA & 45 & 32 & 13 \\
\hline & & AUS/FLUS & CNB & 104 & 82 & 22 \\
\hline & & & FNA & 104 & 75 & 29 \\
\hline Nagarkatti et al. ${ }^{.1}$ (2013) & USA & AUS/FLUS & FNA & 51 & 28 & 23 \\
\hline Oertel et al..$^{32}(2007)$ & USA & Non-diagnostic & FNA & 27 & 23 & 4 \\
\hline \multirow[t]{2}{*}{ Park et al. ${ }^{33}(2011)$} & Korea & Non-diagnostic & CNB & 54 & 53 & 1 \\
\hline & & & FNA & 142 & 73 & 69 \\
\hline Park et al. ${ }^{34}$ (2015) & Korea & AUS/FLUS & FNA & 236 & 155 & 81 \\
\hline \multirow[t]{2}{*}{ Samir et al. ${ }^{35}$ (2012) } & USA & Non-diagnostic & CNB & 69 & 51 & 18 \\
\hline & & & FNA & 69 & 36 & 33 \\
\hline Sullivan et al. ${ }^{36}$ (2014) & USA & AUS/FLUS & FNA & 86 & 48 & 38 \\
\hline Trimboli et al. ${ }^{37}$ (2015) & Italy & Indeterminate & CNB & 198 & 125 & 73 \\
\hline Yeon et al. ${ }^{38}$ (2013) & Korea & Non-diagnostic & CNB & 116 & 108 & 8 \\
\hline Yoon et al. ${ }^{39}$ (2011) & Korea & Non-diagnostic & FNA & 99 & 91 & 8 \\
\hline
\end{tabular}

FNA, fine needle aspiration; CNB, core needle biopsy; AUS/FLUS, atypia/follicular lesion of undetermined significance.

Table 2. Conclusive rates of second CNB and repeat FNA after prior FNA with non-diagnostic or AUS/FLUS significance in thyroid nodules

\begin{tabular}{lcccccr}
\hline & $\begin{array}{c}\text { No. of } \\
\text { subsets }\end{array}$ & $\begin{array}{c}\text { No. of } \\
\text { patients }\end{array}$ & $\begin{array}{c}\text { Fixed effect model } \\
(95 \% \mathrm{Cl})\end{array}$ & $\begin{array}{c}\text { Heterogeneity } \\
(\mathrm{p} \text {-value })\end{array}$ & $\begin{array}{c}\text { Random effect model } \\
(95 \% \mathrm{CI})\end{array}$ & $\begin{array}{c}\text { Egger's } \\
\text { test }\end{array}$ \\
\hline Overall & 35 & 4,566 & $0.690(0.675-0.704)$ & $<.001$ & $0.748(0.701-0.791)$ & .009 \\
Second CNB & 11 & 1,134 & $0.775(0.745-0.802)$ & $<.001$ & $0.879(0.801-0.929)$ & $<.001$ \\
$\quad$ Non-diagnostic & 6 & 533 & $0.897(0.864-0.923)$ & $<.001$ & $0.927(0.847-0.966)$ & .122 \\
$\quad$ AUS/FLUS & 5 & 601 & $0.712(0.671-0.749)$ & $<.001$ & $0.794(0.675-0.877)$ & .030 \\
Repeat FNA & 24 & 3,432 & $0.670(0.653-0.686)$ & $<.001$ & $0.684(0.627-0.736)$ & .651 \\
$\quad$ Non-diagnostic & 11 & 1,739 & $0.674(0.650-0.697)$ & $<.001$ & $0.699(0.596-0.785)$ & .773 \\
$\quad$ AUS/FLUS & 13 & 1,693 & $0.666(0.642-0.689)$ & $<.001$ & $0.673(0.608-0.732)$ & .719 \\
\hline
\end{tabular}

CNB, core needle biopsy; FNA, fine needle aspiration; AUS/FLUS, atypia of undetermined significance/follicular lesion of undetermined significance; Cl, confidence interval. 


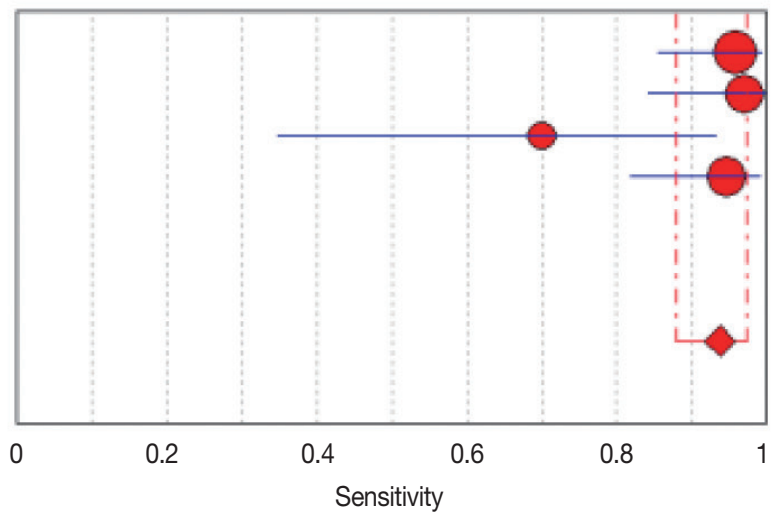

Choi et al..$^{17}$

Choi et al. ${ }^{18}$

Lee et al.26

Yeon et al. 38

Pooled sensitivity $=0.94$ (0.88 to 0.97$)$

Chi-square $=6.44 ; \mathrm{df}=3(\mathrm{p}=.0921)$

Inconsistency $\left(l^{2}\right)=53.4 \%$

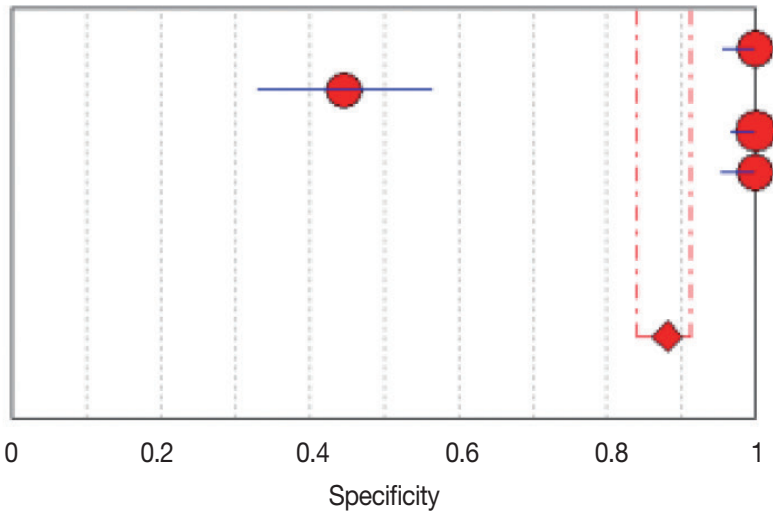

Pooled specificity $=0.88$ ( 0.84 to 0.91$)$

Chi-square $=149.35 ; \mathrm{df}=3(\mathrm{p}=.0000)$

Inconsistency $\left(I^{2}\right)=98.0 \%$

Specificity $(95 \% \mathrm{Cl})$

$1.00(0.96-1.00)$

$0.45(0.33-0.57)$

$1.00(0.97-1.00)$

$1.00(0.95-1.00)$

Fig. 2. The sensitivity (A) and specificity (B) of follow-up core needle biopsy for prediction of papillary thyroid carcinoma after prior fine needle aspiration with non-diagnostic or atypia/follicular lesion of undetermined significance in thyroid nodules.

rate of 0.690 (95\% confidence interval [CI], 0.675 to 0.704 ) and 0.748 (95\% CI, 0.701 to 0.791 ) with the fixed and random effect models, respectively. For follow-up $\mathrm{CNB}$, the conclusive rate was 0.775 (95\% CI, 0.745 to 0.802 ) and 0.879 (95\% CI, 0.801 to 0.929 ) with the fixed and random effect models, respectively. In rFNA, the conclusive rate was 0.670 (95\% CI, 0.653 to 0.686 ) and 0.684 (95\% CI, 0.627 to 0.736 ) with the fixed and random effect models, respectively. The ranges of conclusive rates were $0.631-0.981$ and $0.286-0.942$ for follow-up CNB and rFNA, respectively. Follow-up CNB showed a higher conclusive rate compared with rFNA (OR, 5.707; 95\% CI, 2.530 to 12.875$)$. The heterogeneity of eligible studies was significant in both follow-up CNB and rFNA groups $\left(\mathrm{I}^{2}=90.4 \%, \mathrm{p}<\right.$ 0.001 and $\mathrm{I}^{2}=90.3 \%, \mathrm{p}<0.001$, respectively). In sensitivity analysis, no study had an effect on the concordance rates for either follow-up CNB (range, 0.864 to 0.896 ) or rFNA (range, 0.670 to 0.697 ). Subgroup analysis revealed a significant difference in conclusive rate between follow-up $\mathrm{CNB}$ and rFNA in both non-diagnostic $(0.927 ; 95 \% \mathrm{CI}, 0.848$ to 0.966 vs $0.699 ; 95 \%$ CI, 0.596 to 0.785$)$ and AUSL/FLUS cases $(0.794 ; 95 \%$ CI, 0.675 to 0.877 vs $0.673 ; 95 \% \mathrm{CI}, 0.608$ to 0.732 ) with the random effect model (Table 2).

Egger's test revealed that the follow-up CNB group showed a significant publication bias $(\mathrm{p}=.009)$. Additionally, fail-safe $\mathrm{N}$ and trim-fill tests were performed for confirmation of the degree of publication bias in the CNB group. The number of missing studies that would produce a p-value higher than alpha was 5,401 on the fail-safe $\mathrm{N}$ test. Because there were 11 observed studies, the publication bias was not large. In addition, the trim and fill test showed no significant difference between the observed and adjusted values. Therefore, we concluded that the publication bias in the follow-up CNB group was not significant through interpretation of Egger's test, Begg's funnel plot, the fail-safe $\mathrm{N}$ test, and the trim-fill test. In the rFNA group, there was no significant publication bias according to Egger's test ( $\mathrm{p}=$ .651 ) or Begg's funnel plots.

\section{DTA review of follow-up CNB as a follow-up study}

For confirmation of the diagnostic accuracy of follow-up CNB, we conducted a DTA review. The pooled sensitivity and specificity values were 0.94 (95\% CI, 0.88 to 0.97 ) and 0.88 (95\% CI, 0.84 to 0.91 ), respectively (Fig. 2). The sensitivity and spe- 


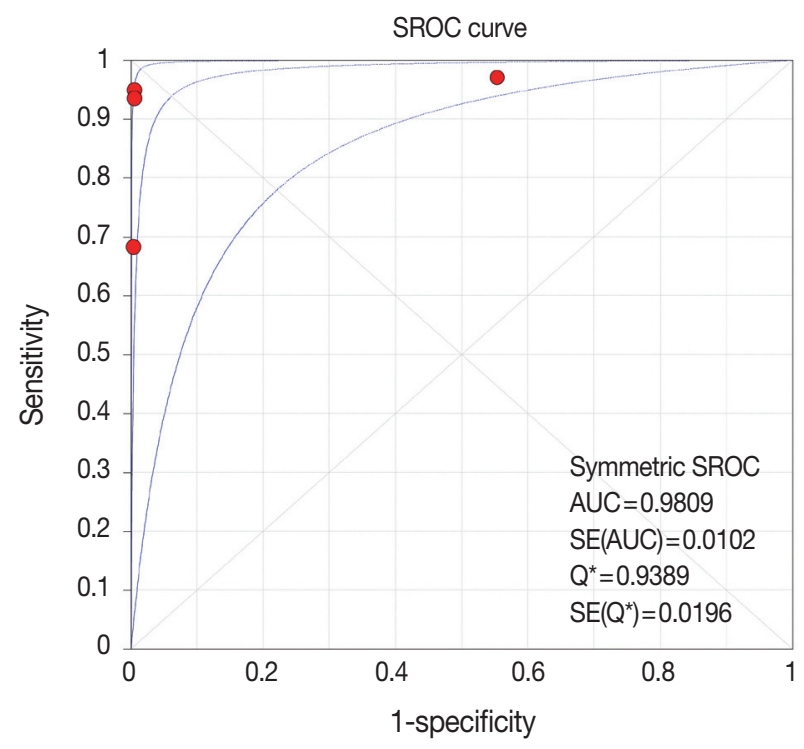

Fig. 3. The summary receiver operating characteristics (SROC) curve of follow-up core needle biopsy for prediction of papillary thyroid carcinoma after prior fine needle aspiration with non-diagnostic or atypia/follicular lesion of undetermined significance in thyroid nodules. AUC, area under the curve.

cificity of eligible studies ranged from 0.70 to 0.97 and from 0.45 to 1.00 , respectively. On the SROC curve, the value of AUC was 0.981 (Fig. 3). In addition, the diagnostic OR was $448.73(95 \%$ CI, 36.63 to $5,497.51$ ).

\section{DISCUSSION}

The current guidelines recommend FNA as the initial test for thyroid nodules. ${ }^{4-6}$ Although rFNA was applied to thyroid nodules with non-diagnostic and AUS/FLUS findings, ${ }^{4-6}$ the effectiveness of rFNA has not been fully elucidated. The present study is the first meta-analysis and DTA review of published studies on the diagnostic role of follow-up CNB compared with rFNA after initial FNA with non-diagnostic or AUS/FLUS results.

In the assessment of thyroid nodule, FNA is the most cost-effective initial test. Recently, FNA using ultrasonography has produced a higher quality of specimens for more precise diagnosis. ${ }^{10}$ However, some cases were non-diagnostic or AUS/FLUS, and the rates of inconclusive results ranged from $10 \%-33.6 \%$ in previous studies. ${ }^{6,8,9,40}$ Although the current guidelines recommend rFNA as a follow-up study for thyroid nodules with initial inconclusive results, ${ }^{4-6} \mathrm{rFNA}$ does not ensure conclusive results. The inconclusive rates of $\mathrm{rFNA}$ in previous studies ranged from $9.9 \%$ to $72.0 \% .^{18,29,34,36}$ In the current meta-analysis, the rate of inconclusive results for $\mathrm{rFNA}$ was $31.6 \%$. If the cellularity of initial FNA is low, rFNA can also show low cellularity. In a pre- vious study, rFNA was the most significant risk factor affecting repeat non-diagnostic results. ${ }^{18}$ This situation might be caused by the nature of the thyroid nodule, including factors such as intratumoral calcification and cystic change. ${ }^{41-43}$ In addition, the quality of the specimen might also be affected by the experience of the operator. Consequently, diagnostic surgery is recommended for thyroid nodule with repeat inconclusive results, and the incidence of diagnostic surgery has been reported as $22.2 \%$ $94.7 \% .{ }^{24,44-46}$ Therefore, to reduce the inconclusive rate, an alternative follow-up study, such as $\mathrm{CNB}$, could be considered for thyroid nodules with inconclusive readings in initial FNA.

Considering the results of a previous meta-analysis, histologic examination using CNB as an initial test might be useful for obtaining conclusive results. ${ }^{47}$ Other previous comparative studies of FNA and $\mathrm{CNB}$ as an initial test have reported that $\mathrm{CNB}$ has a suspected higher specificity, higher positive predictive value, and lower rate of inconclusive results. ${ }^{11,48}$ However, definitive results for the sensitivity and specificity of $\mathrm{CNB}$ as a followup study have not yet been obtained. ${ }^{10,11,47} \mathrm{CNB}$ showed a lower sensitivity for thyroid glands than for other head and neck lesions. ${ }^{49}$ However, the initial test might be more important for achieving higher sensitivity and patient safety. Furthermore, CNB has some limitations, including bleeding, tumor-cell displacement, and difficulty in approaching thyroid nodules in a posterior portion or close to critical structures, including the carotid artery or trachea; these factors limit its use as an initial test. ${ }^{48,49}$ To obtain an adequate specimen for diagnosis, the experience of the operator may be more important for CNB than for FNA. ${ }^{48}$ Whether CNB is appropriate as an initial test for thyroid nodules is not fully understood and could not be determined in the current systematic review. Despite a previous report that found follow-up CNB to have lower non-diagnostic and inconclusive rates than $\mathrm{rFNA}(7.2 \%$ vs $72.0 \%),{ }^{18}$ many previous studies have reported that CNB showed lower sensitivity than FNA. However, the diagnostic accuracy of follow-up CNB has not been fully elucidated. In the current DTA review, the pooled sensitivity and specificity of follow-up CNB were significantly high. For this reason, follow-up CNB after initial FNA might be useful for predicting malignancy and reducing the inconclusive rate in follow-up study.

There were some limitations in the current meta-analysis. First, most included studies were retrospective rather than prospective evaluations. Many thyroid nodules with initial inconclusive results only involved follow-up with ultrasonography. The conclusive and inconclusive rates of rFNA and follow-up CNB might have been affected by such cases. Therefore, cumu- 
lative prospective studies are needed to determine the effectiveness of follow-up procedures. Second, the current study included both non-diagnostic and AUS/FLUS subgroups, and subgroup analysis was performed. However, the DTA review could be not conducted for the rFNA subgroup due to insufficient information from eligible studies. Thus, a comparison of diagnostic accuracy between follow-up CNB and rFNA could not be performed. Third, because the current guidelines recommend initial FNA test for thyroid nodules, ${ }^{4-6}$ the current meta-analysis was performed only for studies with initial FNA. An investigation of the effectiveness of $\mathrm{CNB}$ as an initial test was therefore not conducted in the current study. Fourth, the current study was analyzed for initial non-diagnostic and AUS/FLUS categories. However, additional evaluations, including subgroup analysis for AUS and FLUS results, could not be performed due to lack of information on follow-up CNB from eligible studies. ${ }^{23}$ Fifth, eligible studies used various criteria for nondiagnostic or indeterminate lesion. The rate of inconclusive results of $\mathrm{CNB}$ may have differed from the real value. Further studies are needed to establish guidelines of pathology reporting for thyroid CNB.

In conclusion, the current study showed that follow-up CNB had a higher conclusive rate than rFNA after initial FNA with inconclusive results. In addition, follow-up CNB had greater diagnostic accuracy for prediction of malignancy than rFNA. Additional prospective studies are required to determine standardized application criteria of follow-up CNB in daily practice.

\section{Conflicts of Interest}

No potential conflict of interest relevant to this article was reported.

\section{REFERENCES}

1. Sprague BL, Warren Andersen S, Trentham-Dietz A. Thyroid cancer incidence and socioeconomic indicators of health care access. Cancer Causes Control 2008; 19: 585-93.

2. Enewold L, Zhu K, Ron E, et al. Rising thyroid cancer incidence in the United States by demographic and tumor characteristics, 19802005. Cancer Epidemiol Biomarkers Prev 2009; 18: 784-91.

3. Mercante G, Frasoldati A, Pedroni C, et al. Prognostic factors affecting neck lymph node recurrence and distant metastasis in papillary microcarcinoma of the thyroid: results of a study in 445 patients. Thyroid 2009; 19: 707-16.

4. Cibas ES, Ali SZ. The Bethesda System for Reporting Thyroid Cytopathology. Thyroid 2009; 19: 1159-65.
5. American Thyroid Association (ATA) Guidelines Taskforce on Thyroid Nodules and Differentiated Thyroid Cancer, Cooper DS, Doherty GM, et al. Revised American Thyroid Association management guidelines for patients with thyroid nodules and differentiated thyroid cancer. Thyroid 2009; 19: 1167-214.

6. Gharib H, Papini E, Paschke R, et al. American Association of Clinical Endocrinologists, Associazione Medici Endocrinologi, and European Thyroid Association medical guidelines for clinical practice for the diagnosis and management of thyroid nodules: executive summary of recommendations. J Endocrinol Invest 2010; 33(5 suppl 1): 51-6.

7. Yang J, Schnadig V, Logrono R, Wasserman PG. Fine-needle aspiration of thyroid nodules: a study of 4703 patients with histologic and clinical correlations. Cancer 2007; 111: 306-15.

8. Yassa L, Cibas ES, Benson CB, et al. Long-term assessment of a multidisciplinary approach to thyroid nodule diagnostic evaluation. Cancer 2007; 111: 508-16.

9. Nayar R, Ivanovic M. The indeterminate thyroid fine-needle aspiration: experience from an academic center using terminology similar to that proposed in the 2007 National Cancer Institute Thyroid Fine Needle Aspiration State of the Science Conference. Cancer 2009; 117: 195-202.

10. Harvey JN, Parker D, De P, Shrimali RK, Otter M. Sonographically guided core biopsy in the assessment of thyroid nodules. J Clin Ultrasound 2005; 33: 57-62.

11. Renshaw AA, Pinnar N. Comparison of thyroid fine-needle aspiration and core needle biopsy. Am J Clin Pathol 2007; 128: 370-4.

12. Zamora J, Abraira V, Muriel A, Khan K, Coomarasamy A. MetaDiSc: a software for meta-analysis of test accuracy data. BMC Med Res Methodol 2006; 6: 31.

13. Pyo JS, Sohn JH, Kang G. BRAF immunohistochemistry using clone VE1 is strongly soncordant with BRAF(V600E) mutation test in papillary thyroid carcinoma. Endocr Pathol 2015; 26: 211-7.

14. Anderson TJ, Atalay MK, Grand DJ, Baird GL, Cronan JJ, Beland MD. Management of nodules with initially nondiagnostic results of thyroid fine-needle aspiration: can we avoid repeat biopsy? Radiology 2014; 272: 777-84.

15. Baloch Z, LiVolsi VA, Jain P, et al. Role of repeat fine-needle aspiration biopsy (FNAB) in the management of thyroid nodules. Diagn Cytopathol 2003; 29: 203-6.

16. Chen JC, Pace SC, Chen BA, Khiyami A, McHenry CR. Yield of repeat fine-needle aspiration biopsy and rate of malignancy in patients with atypia or follicular lesion of undetermined significance: the impact of the Bethesda System for Reporting Thyroid Cytopathology. Surgery 2012; 152: 1037-44.

17. Choi YJ, Baek JH, Ha EJ, et al. Differences in risk of malignancy and 
management recommendations in subcategories of thyroid nodules with atypia of undetermined significance or follicular lesion of undetermined significance: the role of ultrasound-guided core-needle biopsy. Thyroid 2014; 24: 494-501.

18. Choi SH, Baek JH, Lee JH, et al. Thyroid nodules with initially nondiagnostic, fine-needle aspiration results: comparison of core-needle biopsy and repeated fine-needle aspiration. Eur Radiol 2014; 24: 2819-26.

19. Dincer N, Balci S, Yazgan A, et al. Follow-up of atypia and follicular lesions of undetermined significance in thyroid fine needle aspiration cytology. Cytopathology 2013; 24: 385-90.

20. Gocun PU, Karakus E, Bulutay P, Akturk M, Akin M, Poyraz A. What is the malignancy risk for atypia of undetermined significance? Three years' experience at a university hospital in Turkey. Cancer Cytopathol 2014; 122: 604-10.

21. Gweon HM, Son EJ, Youk JH, Kim JA. Thyroid nodules with Bethesda system III cytology: can ultrasonography guide the next step? Ann Surg Oncol 2013; 20: 3083-8.

22. Ho AS, Sarti EE, Jain KS, et al. Malignancy rate in thyroid nodules classified as Bethesda category III (AUS/FLUS). Thyroid 2014; 24: 832-9.

23. Hyeon J, Ahn S, Shin JH, Oh YL. The prediction of malignant risk in the category "atypia of undetermined significance/follicular lesion of undetermined significance" of the Bethesda System for Reporting Thyroid Cytopathology using subcategorization and BRAF mutation results. Cancer Cytopathol 2014; 122: 368-76.

24. Jo VY, Vanderlaan PA, Marqusee E, Krane JF. Repeatedly nondiagnostic thyroid fine-needle aspirations do not modify malignancy risk. Acta Cytol 2011; 55: 539-43.

25. Lee SH, Oh HW, Fang Y, et al. Identification of plant compounds that disrupt the insect juvenile hormone receptor complex. Proc Natl Acad Sci U S A 2015; 112: 1733-8.

26. Lee SH, Kim MH, Bae JS, Lim DJ, Jung SL, Jung CK. Clinical outcomes in patients with non-diagnostic thyroid fine needle aspiration cytology: usefulness of the thyroid core needle biopsy. Ann Surg Oncol 2014; 21: 1870-7.

27. Moon HJ, Kim EK, Yoon JH, Kwak JY. Malignancy risk stratification in thyroid nodules with nondiagnostic results at cytologic examination: combination of thyroid imaging reporting and data system and the Bethesda System. Radiology 2015; 274: 287-95.

28. Moslavac S, Matesa-Anić D, Matesa N, Kusić Z. When to repeat thyroid fine needle aspiration cytology? Acta Clin Croat 2012; 51: 549-54.

29. Na DG, Min HS, Lee H, Won JK, Seo HB, Kim JH. Role of core needle biopsy in the management of atypia/follicular lesion of undetermined significance thyroid nodules: comparison with repeat fine-needle aspiration in subcategory nodules. Eur Thyroid J 2015; 4: 189-96.

30. Na DG, Kim JH, Sung JY, et al. Core-needle biopsy is more useful than repeat fine-needle aspiration in thyroid nodules read as nondiagnostic or atypia of undetermined significance by the Bethesda system for reporting thyroid cytopathology. Thyroid 2012; 22: 468-75.

31. Nagarkatti SS, Faquin WC, Lubitz CC, et al. Management of thyroid nodules with atypical cytology on fine-needle aspiration biopsy. Ann Surg Oncol 2013; 20: 60-5.

32. Oertel YC, Miyahara-Felipe L, Mendoza MG, Yu K. Value of repeated fine needle aspirations of the thyroid: an analysis of over ten thousand FNAs. Thyroid 2007; 17: 1061-6.

33. Park KT, Ahn SH, Mo JH, et al. Role of core needle biopsy and ultrasonographic finding in management of indeterminate thyroid nodules. Head Neck 2011; 33: 160-5.

34. Park VY, Kim EK, Kwak JY, Yoon JH, Moon HJ. Malignancy risk and characteristics of thyroid nodules with two consecutive results of atypia of undetermined significance or follicular lesion of undetermined significance on cytology. Eur Radiol 2015; 25: 2601-7.

35. Samir AE, Vij A, Seale MK, et al. Ultrasound-guided percutaneous thyroid nodule core biopsy: clinical utility in patients with prior nondiagnostic fine-needle aspirate. Thyroid 2012; 22: 461-7.

36. Sullivan PS, Hirschowitz SL, Fung PC, Apple SK. The impact of atypia/follicular lesion of undetermined significance and repeat fine-needle aspiration: 5 years before and after implementation of the Bethesda System. Cancer Cytopathol 2014; 122: 866-72.

37. Trimboli P, Nasrollah N, Amendola S, et al. A cost analysis of thyroid core needle biopsy vs. diagnostic surgery. Gland Surg 2015; 4: 307-11.

38. Yeon JS, Baek JH, Lim HK, et al. Thyroid nodules with initially nondiagnostic cytologic results: the role of core-needle biopsy. Radiology 2013; 268: 274-80.

39. Yoon JH, Moon HJ, Kim EK, Kwak JY. Inadequate cytology in thyroid nodules: should we repeat aspiration or follow-up? Ann Surg Oncol 2011; 18: 1282-9.

40. Degirmenci B, Haktanir A, Albayrak R, et al. Sonographically guided fine-needle biopsy of thyroid nodules: the effects of nodule characteristics, sampling technique, and needle size on the adequacy of cytological material. Clin Radiol 2007; 62: 798-803.

41. Choi YS, Hong SW, Kwak JY, Moon HJ, Kim EK. Clinical and ultrasonographic findings affecting nondiagnostic results upon the second fine needle aspiration for thyroid nodules. Ann Surg Oncol 2012; 19: 2304-9.

42. Choi $\mathrm{SH}$, Han $\mathrm{KH}$, Yoon JH, et al. Factors affecting inadequate sampling of ultrasound-guided fine-needle aspiration biopsy of thyroid nodules. Clin Endocrinol (Oxf) 2011; 74: 776-82.

43. Moon HJ, Kwak JY, Kim EK, Kim MJ. Ultrasonographic character- 
istics predictive of nondiagnostic results for fine-needle aspiration biopsies of thyroid nodules. Ultrasound Med Biol 2011; 37: 549-55.

44. Orija IB, Piñeyro M, Biscotti C, Reddy SS, Hamrahian AH. Value of repeating a nondiagnostic thyroid fine-needle aspiration biopsy. Endocr Pract 2007; 13: 735-42.

45. Hryhorczuk AL, Stephens T, Bude RO, et al. Prevalence of malignancy in thyroid nodules with an initial nondiagnostic result after ultrasound guided fine needle aspiration. Ultrasound Med Biol 2012; 38: 561-7.

46. Lubitz CC, Nagarkatti SS, Faquin WC, et al. Diagnostic yield of nondiagnostic thyroid nodules is not altered by timing of repeat biopsy.
Thyroid 2012; 22: 590-4.

47. Li L, Chen BD, Zhu HF, et al. Comparison of pre-operation diagnosis of thyroid cancer with fine needle aspiration and core-needle biopsy: a meta-analysis. Asian Pac J Cancer Prev 2014; 15: 7187-93.

48. Yoon JH, Kim EK, Kwak JY, Moon HJ. Effectiveness and limitations of core needle biopsy in the diagnosis of thyroid nodules: review of current literature. J Pathol Transl Med 2015; 49: 230-5.

49. Novoa E, Gürtler N, Arnoux A, Kraft M. Role of ultrasound-guided core-needle biopsy in the assessment of head and neck lesions: a meta-analysis and systematic review of the literature. Head Neck 2012; 34: 1497-503. 